\title{
RESILIENCIA Y ANSIEDAD EN MADRES DE MENORES QUE PADECEN DIABETES TIPO 1
}

\author{
Antonio Zayas García \\ Paloma Gil-Olarte Márquez \\ Cristina Guerrero Rodríguez \\ Rocío Guil Bozal \\ Universidad de Cádiz \\ Email: antonio.zayas@uca.es \\ https://doi.org/10.17060/ijodaep.2017.n1.v3.982
}

Fecha de Recepción: 5 Febrero 2017

Fecha de Admisión: 1 Abril 2017

\section{RESUMEN}

La diabetes tipo 1 es una de las enfermedades crónicas más comunes en la infancia y la adolescencia según la American Diabetes Association (2009). Requiere un tratamiento complejo que implica la atención constante por parte de los cuidadores principales, que suelen ser las madres (Garcia-Campayo, Sanz-Carrillo, \& Tazon-Ansola, 2009). Esto a su vez supone una sobrecarga que determina el bienestar psicológico de estas cuidadoras, siendo muy frecuente la manifestación de sintomatología ansiosa. Nuestros objetivos con el presente trabajo es conocer el nivel de ansiedad estado/rasgo en este colectivo, estudiar sus niveles de resiliencia y analizar la relación y la posible capacidad explicativa y/o predictiva que la resiliencia pueda tener sobre los niveles de ansiedad en estas madres. Para llevar a cabo este trabajo se ha seleccionado un total de 54 madres de niños que padecen la enfermedad, con una media de edad de 42,40 años (DT=5,1), de la Asociación de Diabéticos de Jerez (ADIJE). Para la evaluación de las variables se ha administrado el State Trait Anxiety Inventory (STAI) (Spielberger, Gorsuch, \& Lushene, 1970, 1982) y la Escala de Resiliencia de Wagnild y Young (1988). Los resultados arrojan niveles medios-altos de ansiedad estado y altos de ansiedad rasgo en las madres. Asimismo, muestran niveles moderados de resiliencia. Respecto a la relación entre la resiliencia y la ansiedad se ha hallado que la dimensión Ecuanimidad de la variable Resiliencia tiene una capacidad predictiva en los niveles de ansiedad estado, y la dimensión Confianza en sí mismo presenta una capacidad predictiva en los niveles de ansiedad rasgo. Concluimos con la importancia de fomentar la resiliencia en madres de menores con diabetes tipo 1 al fin de prevenir la sintomatología ansiosa, así como consideramos necesario continuar estudiando en profundidad las variables psicosociales que puedan evitar la aparición de cuadros psicopatológicos en esta población.

Palabras claves: diabetes infantil, cuidadores informales, ansiedad y resiliencia. 


\section{ABSTRACT}

\section{Resilience and anxiety in mothers of children with type 1 diabetes}

Type 1 diabetes is one of the most common chronic diseases in childhood and adolescence according to the American Diabetes Association (2009). This disease requires complex treatment, which involves a constant attention from children's caretakers, especially their mothers (GarciaCampayo, Sanz-Carrillo, \& Tazon-Ansola, 2009). This attention implies an overload for the mother that involves a risk for the mother's wellbeing. Type-1-diabetes children (T1DC) increase anxiety simptoms among their mothers. Hence, this work aims to know the level of both state/trait anxiety and resilience of mothers with T1DC; and the predictive relationship between resilience and anxiety of mother's T1DC. A sample of 54 mothers with mean age $42,40(S D=5,1)$, of T1DC from the Association of Diabetics of Jerez (ADIJE, Spain) filled the State Trait Anxiety Inventory (STAI) (Spielberger, Gorsuch, \& Lushene, 1970, 1982) and the Wagnild and Young Resilience Scale (1988). Outcomes showed that the participants presented medium-high levels of state anxiety and high trait anxiety. Mother's T1DC scored moderate levels of resilience. Regarding the relationship between resilience and anxiety, Equanimity predicted a negative relationship to state anxiety levels, Confidence was negative related to trait anxiety levels. This work suggests that promoting resilience in mothers with T1DC can help to manage the anxiety symptoms among mothers. However, it is necessary to increase studies that include psychosocial variables that may would be involved in the anxiety regulation abilities of mothers with T1DC.

Keywords: childhood diabetes, informal caregivers, anxiety, and resilience.

\section{ANTECEDENTES}

La diabetes tipo 1 es la enfermedad crónica pediátrica más frecuente según la American Diabetes Association (2009).

Se trata de una enfermedad crónica que provoca un desorden endocrino, caracterizándose por una destrucción de las células pancreáticas betas, que tienen como misión la producción de insulina, esencial para el adecuado metabolismo de la glucosa (Rovet \& Fernández, 1999). El tratamiento de esta enfermedad es complejo y requiere de inyecciones diarias de insulina, controles rutinarios del nivel de glucosa, hábitos dietéticos específicos y realización de ejercicio físico, al fin de mantener los niveles de glucosa lo más ajustado posible a la normoglucemia y con un adecuado control metabólico (Johnson, 1998). Es decir, para el cuidado de esta patología se necesita de un tratamiento continuado, que requiere de unos hábitos y conductas que aseguren la adherencia al tratamiento (Arenas-Bermúdez, Muela Martínez \& García León, 2007).

El padecimiento de esta enfermedad en niños y adolescentes suele ir acompañado por un cambio en sus hábitos cotidianos y de vida, en el que se involucra toda la familia, y por ende a los padres como responsables del cuidado y control de dicha enfermedad (Beléndez, 2012). Es tal la participación de los miembros de la familia que requiere el tratamiento de la diabetes tipo 1 que ha llevado a los autores a conceptualizarla como una enfermedad "compartida", la familia siente la enfermedad como propia (Beveridge, Berg, Wiebe \& Palmer, 2006; Cardona, Marín, Montoya-Castilla \& PradoGascó, 2014).

El adecuado control de esta enfermedad previene o retrasa complicaciones a corto y largo plazo, mejorando la calidad de vida (Cardona et al., 2014). La tensión provocada por el miedo y preocupación a la aparición de complicaciones es bastante frecuente en los cuidadores, generando en ellos una situación de malestar psicológico y ansiedad (Beléndez, Hidalgo, Bermejo, Ros \& Méndez, 2001; Ferrer-Pérez, 2010).

Así, el estrés provocado en los padres por el impacto del diagnostico, las modificaciones que requieren la nueva situación en sus hábitos cotidianos por el tratamiento y las tareas específicas de 
cuidado hacia sus hijos tiene una repercusión negativa en su propio bienestar psicológico (Beléndez, 2012; Jaser, Wittemore, Ambrosino, Lindemann \& Grey, 2009; Streisand, Swit, Wickmarck, Chen \& Holmes, 2005; Zayas, Guil, Mestre, Guerrero \& Gil-Olarte, 2016).

Normalmente la responsabilidad suele recaer sobre una persona, el cuidador principal, de forma privada, no remunerada y en el ámbito doméstico, así como las cargas de tipo emocional y/o social (Horsch, McManus \& Kennedy, 2008; Seguí, Ortiz-Tallo \& De Diego, 2008). La sobrecarga en este cuidador puede tener una repercusión sobre su nivel de ansiedad y depresión, así como en su calidad de vida, dando lugar a lo que se conoce como el síndrome del cuidador o la sobrecarga del cuidador (López et al., 2009). Numerosos estudios ponen de manifiesto que en el caso de niños con diabetes tipo 1, los cuidadores principales suelen ser las madres (Garcia-Campayo, Sanz-Carrillo \& Tazon-Ansola, 2009).

Entre las diferentes dificultades con las que se va a encontrar el cuidador, la más recurrente puede ser la ansiedad, pudiendo generar una inadecuada adaptación al tratamiento por parte del niño, malestar familiar, e inadecuado control metabólico (Whittemore, Jaser, Chao, Jang \& Grey, 2012).

A pesar de la importancia que posee la ansiedad de las cuidadoras principales de niños diabéticos, no ha recibido la importancia que debiera (Butwickaa, Zalepac, Fendlerd, Szadkowskad \& Mlynarskid, 2013). Parecen existir pocos estudios que midan como afecta la ansiedad en las madres de niños diabéticos. Sin embargo, detectar y tratar a tiempo los trastornos psicológicos en estas madres parecen tener un efecto positivo en el tratamiento de la diabetes (Butwickaa et al., 2013).

En las últimas décadas ha aumentado el interés científico por el impacto que las enfermedades crónicas tienen en quien la padece y en sus familiares (Seguí et al., 2008; Whittemore et al., 2012), centrándose en el efecto en el cuidador principal. Si bien, la mayoría de estudios sobre cuidadores informales se han centrado concretamente en cuidadores de personas con Alzheimer (Seguí et al., 2008). No obstante, siguiendo a estos autores, en los últimos tiempos se ha comenzado a prestar interés a padres de niños con enfermedades crónicas.

Por otro lado, uno de los procesos psicosociales que más interés está generando en las últimas décadas y que guarda relación con el malestar psicológico es la resiliencia (Zayas et al., 2016). Aguila (2000) la define como la capacidad que poseen las personas para resistir, sobreponerse y salir de manera exitosa tras haber pasado por dificultades 0 acontecimientos traumáticos. La resiliencia fomenta y promueve la salud mental, numerosas personas, aun habiendo experimentado un acontecimiento traumático, han conseguido encajarlo y seguir desenvolviéndose y viviendo, incluso en un nivel superior, como si el trauma experimentado hubiese despertado esos recursos latentes e insospechados que antes de experimentar el acontecimiento estresante no habían percibido (Bowen, Morasca \& Meischke, 2003; Vázquez \& Castilla, 2007).

Si bien es cierto que cada vez hay un incremento mayor por estudiar la resiliencia como protectores del desarrollo de cuadros psicopatológicos, como es el caso de la ansiedad, resulta difícil encontrar trabajos sobre dicha relación en padres de niños con diabetes tipo 1 (Zayas et al., 2016).

\section{OBJETIVOS DE LA INVESTIGACIÓN}

El ánimo del presente trabajo surge de la inquietud por profundizar en el conocimiento del malestar psicológico de madres cuidadoras principales de menores con diabetes tipo 1 y la posible relación con aspectos psicosociales como la Resiliencia.

Por ello, los objetivos específicos que se contemplan en el presente estudio son los siguientes:

Conocer los niveles de Ansiedad Estado/Rasgo en madres de menores con diabetes tipo 1.

Estudiar el nivel de Resiliencia en madres de menores con diabetes tipo 1. 
Analizar la relación entre los niveles de Resiliencia y Ansiedad Estado/Rasgo en esta población.

Analizar la posible capacidad explicativa y/o predictiva de los niveles de Resiliencia, en cuanto a los de Ansiedad Estado/Rasgo en este colectivo.

\section{MUESTRA Y/O PARTICIPANTES}

Al fin de poder llevar a cabo este estudio, se ha seleccionado una muestra formada por 54 mujeres, cuyos hijos padecen diabetes tipo 1. Las participantes pertenecen a la Asociación de Diabéticos de Jerez (ADIJE). La edad media es de 42,40 años (34-52), (DT= 5,1), siendo la edad media de los hijos con diabetes de 4,6 años (1-17), (DT=4,6).

Respecto al estado civil encontramos que el 1,9\% de la muestra son solteras, el 88,9\% están casadas, el 7,4\% están divorciadas, y el 1,9\% son viudas.

En cuanto al nivel de estudios, el 37\% poseen estudios primarios, el 35,2\% estudios secundarios (formación profesional y bachillerato) y el $27,8 \%$ estudios superiores.

Por último, referente a la situación laboral, encontramos que el 44,4\% están en situación activa, el $51,9 \%$ se encuentran en situación de desempleo, y el 3,7\% son pensionistas.

\section{METODOLOGía Y/O INSTRUMENTOS UTILIZADOS}

Los criterios de inclusión que se tuvieron en cuenta para seleccionar a las personas que participaron en este estudio, fueron: ser mujer; tener al menos un hijo o hija con diabetes tipo 1 del que fuera la cuidadora principal, que llevara al menos un año con la enfermedad; disponer de un nivel cognitivo que les permitiese comprender de manera adecuada las pruebas que se les administró en el presente estudio; no encontrarse, en el momento de la evaluación, en tratamiento psiquiátrico y/o psicológico debido al padecimiento de un trastorno mental grave; no presentar ninguna patología grave 0 incapacitante.

Las participantes de este estudio fueron reclutadas en el Colegio de Educación Infantil y Primaria Antonio de Nebrija (Jerez de la Frontera), donde se encontraban en una reunión organizada por ADIJE y miembros del Grupo de Investigación HUM-843 Inteligencia Emocional de la Universidad de Cádiz, para explicarles un proyecto de investigación e intervención que se pondrá en marcha con ellas. Tras finalizar la reunión, se les explicó detalladamente el presente estudio, y los objetivos del mismo, para posteriormente pasarles el consentimiento informado, que tuvieron la oportunidad de leer y firmar de manera voluntaria. Pasaron a cumplimentar las pruebas de manera autoadministradas, aunque en presencia del evaluador para la aclaración de cualquier duda que les pudiera surgir.

La información obtenida fue introducida y analizada mediante el programa estadístico SPSS V.22.0.

Para completar la evaluación se administraron los siguientes instrumentos:

Cuestionario de elaboración propia: donde se recogieron datos sociodemográficos, como la edad, estado civil, nivel de estudios, situación laboral, si padecían o no trastorno psicológico o psiquiátrico, o si estaban o habían estado en tratamiento psiquiátrico o psicológico, tiempo que llevaba el menor con la enfermedad, y la edad de éste en el momento de la evaluación.

Inventario de Ansiedad Estado-Rasgo (STAI-E, STAI-R), para la evaluación de la Ansiedad Estado/Rasgo en su adaptación española de TEA llevada a cabo por Seisdedos en 1988, del State Trait Anxiety Inventory (STAI) (Spielberger, Gorsuch \& Lushene, 1970, 1982). Comprende dos escalas separadas de autoevaluación que miden dos dimensiones independientes de la ansiedad: Estado (STAI/E) y Rasgo (STAI/R). La escala E consta de veinte ítems cada una con las que el sujeto puede describir cómo se siente "en un momento particular", mientras que la escala R, también con 20 ítems, puede mostrar cómo se siente la persona "generalmente". Los ítems se valoran en una esca- 
la likert de 0 a 3 puntos, por lo que las puntuaciones en Ansiedad Estado/Rasgo pueden variar desde un mínimo de 0 puntos hasta un máximo de 60. En cuanto a las dimensiones que mide este cuestionario hacen referencia a:

Ansiedad-estado: condición emocional transitoria del organismo humano caracterizada por sentimientos subjetivos conscientemente percibidos, de tensión y aprensión, así como un aumento de la actividad del sistema nervioso autonómico. Un ejemplo de ítem sería: "En este momento me siento nervioso". En la muestra del presente trabajo la fiabilidad mostrada en esta dimensión es de de Cronbach= 0,63.

Ansiedad-rasgo: relativa propensión ansiosa por la que difieren los sujetos en su tendencia a percibir las situaciones como amenazadoras y a elevar, consecuentemente su ansiedad estado (Caci, Baylé, Dossios, Robert \& Boyer, 2003). Un ejemplo de ítem podría ser: "En general siento ganas de llorar". En la muestra de esta investigación esta dimensión ha mostrado una fiabilidad de de Cronbach $=0.72$.

Para la evaluación de la resiliencia se administró la Escala de Resiliencia de Wagnild y Young (1993), en su adaptación al castellano por Novella (2002). El cuestionario se compone de 25 ítems evaluados mediante una escala de 7 puntos, donde 1 es en desacuerdo, y un máximo de acuerdo es 7. Los participantes han de indicar el grado de conformidad con el ítem, ya que todos son calificados positivamente, el rango de puntaje varía entre 25 y 175 (Wagnild \& Young, 1993). Además de proporcionar una medida global de Resiliencia, aporta información de 5 dimensiones que forman parte de la misma, como son:

Ecuanimidad: un ejemplo de ítem sería: "Rara vez me pregunto cuál es la finalidad de todo", en el presente trabajo esta dimensión presenta una fiabilidad de de Crobach=0,61.

Perseverancia: un ejemplo de ítems sería "Cuando planeo algo lo realizo", en la presente investigación muestra una fiabilidad de de Cronbach=0,72.

Confianza en sí mismo: por ejemplo, "Me siento orgulloso de haber logrado cosas en mi vida", la fiabilidad de esta dimensión en este estudio es de de Cronbach: 0,80.

Satisfacción Personal: por ejemplo, "Por lo general encuentro algo de lo que reírme", en esta dimensión la fiabilidad es de de Cronbach: 0,64.

Sentirse bien Solo: por ejemplo, "Puedo estar solo si tengo que hacerlo", esta dimensión no ha podido ser contemplada en la presente investigación, dado que presenta una fiabilidad de de Cronbach: 0,13 .

La puntuación de Resiliencia Total, en el presente trabajo presentó una fiabilidad de de Cronbach: 0,9.

Para el análisis de los resultados obtenidos en esta prueba, no se ha podido tener en cuenta la dimensión "Sentirse bien solo" dado que presentaba un índice de fiabilidad que no es aceptable estadísticamente.

En estudios llevados a cabo por Novella (2002) se demostró la validez concurrente por altos índices de correlación con mediciones bien establecidas de constructos vinculados con la resiliencia.

\section{RESULTADOS}

Para dar respuesta a nuestro primer objetivo calculamos la media de la puntuación directa obtenida en Ansiedad Estado/Rasgo. Posteriormente, teniendo en cuenta el baremo de la prueba, obtuvimos los percentiles a los que correspondían cada una de las puntuaciones directas.

La media en Ansiedad Estado es de 27,77 (DT=11,86) lo cual corresponde a un Percentil entre 65 y 70, correspondiéndose con una puntuación media-alta. Respecto a la Ansiedad Rasgo, la media 
es de 32,37 (DT=10,93) que corresponde a un Percentil=75, lo cual indica altos niveles de Ansiedad Rasgo.

En cuanto a nuestro segundo objetivo, calculamos la media de la puntuación directa que habían obtenido en la Escala de Resiliencia, siendo ésta de 131,30. Atendiendo al baremo de la escala, esta puntuación corresponde con una moderada capacidad de Resiliencia. A la luz de estos resultados podemos inferir que las madres cuidadoras de niños con diabetes tipo 1, presentan niveles moderados de capacidad para resistir a la adversidad y salir fortalecidas de la misma.

Al fin de dar respuesta a nuestro objetivo tres, llevamos a cabo un análisis de correlación bivariada entre las variables Resiliencia (y las dimensiones que la componen) y la Ansiedad Estado/Rasgo.

Tabla 1.

Correlaciones bivariadas entre la Resiliencia y las distintas dimensiones de ésta y la Ansiedad Estado/Ansiedad Rasgo

\begin{tabular}{lll}
\hline Resiliencia y subescalas & Ansiedad Estado & Ansiedad Rasgo \\
\hline Resiliencia &,$- 465^{* *}$ &,$- 662^{* *}$ \\
Satisfacción Personal & $-376^{* *}$ &,$- 511^{* *}$ \\
Ecuanimidad &,$- 439^{* *}$ &,$- 564^{* *}$ \\
Confianza en sí mismo &,$- 372^{* *}$ &,$- 606^{* *}$ \\
Perseverancia &,$- 366^{* *}$ &,$- 534^{* *}$ \\
\hline
\end{tabular}

Como podemos observar en la Tabla 1, tanto la variable Resiliencia globalmente considerada, como las dimensiones Satisfacción Personal, Ecuanimidad, Confianza en sí mismo y Perseverancia muestran una correlación negativa estadísticamente significativa con los niveles de ansiedad estado y rasgo. Lo cual nos indica que cuanto mayor son las puntuaciones en niveles de Resiliencia, menores puntuaciones obtienen en niveles de Ansiedad Estado/Rasgo.

Para dar respuesta a nuestro último objetivo y comprobar si las correlaciones encontradas implican, a su vez que la Resiliencia (con sus diferentes dimensiones) explican y predicen los niveles de Ansiedad Estado/Rasgo en nuestra muestra, realizamos dos análisis de regresión lineal. En el primero se toma como variable dependiente la Ansiedad Estado y como variable predictora la Resiliencia (con sus diferentes dimensiones) cuyos resultados mostramos en la tabla 2.

Tabla 2.

Resumen del análisis de regresión efectuado

\begin{tabular}{llll}
\hline Variable /variable predictor & $\% R^{2}$ & $F$ & B \\
\hline Ansiedad Estado & $19,2 \%$ & $(5,53) 13,577$ (sig.001) & \\
• Ecuanimidad (Resiliencia) & & & -.439 (sig .001)
\end{tabular}


La Ansiedad Estado es explicada en un $19,2 \%$ por la variable Resiliencia, compuesta por las dimensiones Satisfacción Personal, Ecuanimidad, Confianza en sí mismo y Perseverancia. Sin embargo, como hemos reflejado en la tabla 2, únicamente muestra una capacidad predictiva significativa la dimensión Ecuanimidad, mostrando una Beta ( ) negativa de -,439. Este dato nos indica que si incrementamos la Ecuanimidad en las participantes de nuestra muestra, es decir su capacidad para tener una perspectiva equilibrada de su propia vida y experiencias, así como el que se tomen la adversidad de forma lo más tranquilamente posible, disminuiríamos sus niveles de Ansiedad Estado, es decir, su respuesta ansiosa ante determinadas situaciones puntuales, como puede ser una hipoglucemia.

En el segundo análisis de regresión lineal, la variable dependiente es la Ansiedad Rasgo, y nuevamente como predictora la Resiliencia (con sus diferentes dimensiones), en la tabla 3 podemos observar los resultados obtenidos:

Tabla 3.

Resumen del análisis de regresión efectuado

\begin{tabular}{llll}
\hline Variable /variable predictor & $\% R^{2}$ & $F$ & B \\
\hline Ansiedad Rasgo & $36,7 \%$ & $(1,57)$ 33,066 (sig.000) & \\
$\begin{array}{l}\text { Confianza en sí mismo } \\
\text { (Resiliencia) }\end{array}$ & & &,- 606 (sig .000)
\end{tabular}

En este caso nos encontramos que la Ansiedad Rasgo es explicada en un $36,7 \%$ por la variable Resiliencia, y sus dimensiones: Satisfacción Personal, Ecuanimidad, Confianza en sí mismo y Perseverancia. Si bien, como hemos reflejado en la tabla 3, únicamente muestra una capacidad predictiva estadísticamente significativa la dimensión Confianza en sí mismo, mostrando una Beta ( ) negativa de -,606. Con estos resultados podemos inferir que si incrementamos la Confianza en sí misma, en estas madres, podríamos disminuir la tendencia general a percibir las situaciones como amenazadoras, disminuyendo así los niveles de ansiedad general.

Por lo que estos resultados no sólo nos han permitido conocer la influencia que tiene la Resiliencia sobre los niveles de Ansiedad Estado/Rasgo además, nos ha permitido afinar más los resultados, indicándonos que dimensión de la primera explica y predice los niveles de la segunda, datos que consideramos de relevante importancia de cara a plantear programas de intervención psicológica con este colectivo.

\section{DISCUSIÓN}

Los resultados obtenidos arrojan la existencia de sintomatología ansiosa en las madres de niños con diabetes tipo 1, siendo mayor el nivel de Ansiedad Rasgo. Eso quiere decir que estas madres tienen la tendencia a vivir las situaciones como amenazantes, y los diferentes acontecimientos alteran y aumentan sus niveles de ansiedad. Ello puede estar explicado por lo que señalan Beléndez et al. (2001) sobre la tensión que provoca el miedo a la aparición de complicaciones derivadas de la diabetes que padecen sus hijos, lo cual se ha podido cronificar a lo largo de los meses desde el diagnostico.

Respecto a los niveles de Resiliencia, las madres que forman nuestra muestra se presentan moderadamente resilientes, lo cual tiene que ver con el hecho de estar enfrentándose a diario a 
situaciones adversas probablemente relacionadas con la enfermedad de sus hijos y el tratamiento de la misma. Ante estas situaciones salen fortalecidas, puesto que comprueban que, pese al sufrimiento y resistencia, tienen capacidad para afrontarlo de manera exitosa.

Por último, en cuanto a la relación entre la Resiliencia y la Ansiedad, los resultados arrojan que de cara a plantear programas de intervención psicológica, el fomentar que las madres consigan una visión más equilibrada de las circunstancias adversas y tomen los acontecimientos con actitudes más positivas, podría reducir los niveles de Ansiedad Estado, ante situaciones puntuales, como episodios de hipoglucemia. Asimismo, nuestro estudio nos aporta información respecto a la importancia de fomentar en las madres el que crean en sus habilidades y capacidades para afrontar las situaciones, para disminuir la Ansiedad Rasgo. Estos resultados van en la misma línea que lo propuesto por Bowen et al. (2003) o por Vázquez y Castilla (2007) quienes señalan la Resiliencia como predictora de bienestar psicológico.

\section{CONCLUSIONES}

A la luz de los resultados obtenidos mediante el presente estudio podemos concluir que:

Las madres de menores con diabetes tipo 1 presentan niveles medio-alto de Ansiedad Estado y niveles elevados de Ansiedad Rasgo.

Las mujeres que forman parte de nuestra muestra presentan niveles moderados de Resiliencia.

La Resiliencia, concretamente en su dimensión Ecuanimidad predice los niveles de Ansiedad Estado.

La dimensión de Confianza en sí mismo de la variable Resiliencia predice los niveles de Ansiedad Rasgo.

Los resultados obtenidos en el presente estudio aportan información relevante, de cara a la elaboración de programas de intervención psicológica con esta población.

Se hace necesario el continuar profundizando en el conocimiento de aquellos factores psicosociales que pueden prevenir el desarrollo de cuadros psicopatológicos como son trastornos de ansiedad en madres de menores que padecen diabetes tipo 1.

\section{REFERENCIAS BIBLIOGRÁFICAS}

Aguila, M. (2000). Diferencia en la resiliencia según género y nivel socioeconómico en adolescentes. Lima: Universidad Nacional de Federico Villareal.

American Diabetes Association. (2009). Standars of medical care in diabetes. Diabetes Care, 32, 1361.

Arenas-Bermúdez, C., Muela-Martínez, J. A. \& García-León, A. (2007). Relación entre adherencia objetiva al tratamiento en la diabetes infantil y variables psicológicas de los cuidadores. Index de Enfermería, 16(58), 16-20.

Beléndez, M, Hidalgo, M. D, Bermejo, R, Ros, M. C. \& Méndez, F. J. (2001). Psychometric properties of the perceived stress inventory in diabetes care: a pilot study with a sample of parents of children with insulin-dependent diabetes. Journal of the Ibero-American Association of Diagnosis and Psychological Evaluation, 11, 9-27.

Beléndez, M. (2012). Estrategias de afrontamiento y bienestar emocional en padres y madres de niños y adolescentes con diabetes: diferencias de género. Ansiedad y Estrés, 18 (2/3), 177-186.

Beveridge, R. M., Berg, C. A., Wiebe, D. J. \& Palmer, D. L. (2006). Mother and Adolescent Representations of Illness Ownership and Stressful Events Surrounding Diabetes. Journal of Pediatric Psychology, 31(8), 818-827.

Bowen, D. J., Morasca, A. A. \& Meischke, H. (2003). Measures and Correlates of Resilience. Women y Health, 38(2), 65-76. 
Butwickaa, A., Zalepac, A., Fendlerd, W., Szadkowskad, A. \& Mlynarskid, W. (2013). Maternal depressive symptoms predict acute hospitalization among children with type 1 diabetes. Pediatric Diabetes, 14(4), 288-294.

Caci, H., Baylé, F., Dossios, C. Robert, P. \& Boyer, P. (2003). The Spielberger Trait Anxiety Inventory measures more than anxiety. European Psychiatry, 18, 394-400.

Cardona, A. G., Marín, M. P., Montoya-Castilla, I. \& Prado-Gascó, V. J. (2014). Ansiedad en cuidadoras principales de niños con diabetes mellitus Tipo I. Calidad de vida y salud, 7(2).

Ferrer-Pérez, J. (2010). Ansiedad y respiración diafragmática. Enfermería Integral, 89, 58-60.

Garcia-Campayo, J., Sanz-Carrillo, C. \& Tazon-Ansola, P. (2009). La adaptación y el afrontamiento a la enfermedad. En M. Mompart (Ed.), Relación y comunicación (2 $2^{\mathrm{a}}$ ed, pp. 187-189). Madrid: DAE.

Horsch, A., McManus, F. \& Kennedy, P. (2008). Cognitive and Non-Cognitive Factors Associated with Posttraumatic Stress Symptoms in Mothers of Children with Type 1 Diabetes. Behavioural and Cognitive Psychotherapy, 40(4), 400-411.

Jaser, S. S., Whittemore, R., Ambrosino, J. M., Lindemann, E. \& Grey, M. (2009). Coping and psychosocial adjustment in mothers of young children with type 1 diabetes. Children's Health Care, 38(2), 91-106.

Johnson, S. (1998). Diabetes mellitus in childhood. En D.K. Routh, Handbook of Pediatric psychology. pp. 1-31. New York: The Guilford Press.

López, M., Orueta, M., Gómez, S., Sánchez, A., Carmona, J. \& Alonso, F. (2009). El rol del cuidador de personas dependientes y sus repercusiones sobre su calidad de vida y salud. Revista Clínica de Medicina de Familia, 2(7), 332-334.

Novella, A. (2002). Incremento de la resiliencia luego de la aplicación de un programa de psicoterapia breve en madres adolescentes. Tesis para optar el grado de Magíster en Psicología, mención en Psicología clínica y de la salud. Universidad Nacional Mayor de San Marcos, Lima, Perú.

Rovet, J. \& Fernández, C. (1999). Insulin-Dependent Diabetes Mellitus. En R.T. Brown (Ed.) Cognitive aspects of chronic illness in children (pp.143-171). New York: The Guilford Press.

Seguí, J., Ortiz-Tallo, M. \& De Diego, Y. (2008). Factores asociados al estrés del cuidador primario de niños con autismo: Sobrecarga, psicopatología y estado de salud. Anales de psicología, $1(24), 100-105$.

Seisdedos, N. (1988). Adaptación Española del STAl, Cuestionario de ansiedad estado-rasgo Spanish adaptation of the STAl, State-Trait Anxiety Inventory. Madrid: Tea Ediciones.

Spielberger, C. D., Gorsuch, R. \& Lushene, R. (1970). Manual for the State-Trait Anxiety Inventory. Palo Alto, California: Consulting Psychologist Press.

Spielberger, C. D., Gorsuch, R. L. \& Lushene, R. (1982). Manual del Cuestionario de Ansiedad Estado/Rasgo (STAI). Madrid, España: TEA Ediciones.

Streisand, R., Swift, E., Wickmark, T., Chen, R. \& Holmes, C. S. (2005). Pediatric parenting stress among parents of children with type 1 diabetes: the role of self-efficacy, responsibility, and fear. Journal of pediatric psychology, 30(6), 513-521.

Vázquez, C. \& Castilla, C. (2007). Emociones positivas y crecimiento postraumático en el cáncer de mama. Psicooncología, 4(2-3), 385-404.

Wagnild, G. \& Young, H. (1993). Development and psychometric evaluation of the resilience scale. Journal of Nursing Measurement, 1(2), 165-167.

Whittemore, R., Jaser, S., Chao, A., Jang, M. \& Grey, M. (2012). Psychological experience of parents of children with type 1 diabetes: a systematic mixed-studies review. The Diabetes Educator, 38(4), 562-579. 
Zayas, A., Guil, R., Guerrero, C., Gil-Olarte, P. \& Mestre, J. M. (2016, December). Resilience, optimism, and depression in caregivers of diabetic children. In QUAESTI-Virtual Multidisciplinary Conference (No. 1). 